\title{
Video Article \\ Chitosan/Interfering RNA Nanoparticle Mediated Gene Silencing in Disease Vector Mosquito Larvae
}

\author{
Xin Zhang ${ }^{* 1}$, Keshava Mysore ${ }^{\star^{2,3}}$, Ellen Flannery ${ }^{3,4}$, Kristin Michel ${ }^{1}$, David W. Severson ${ }^{3,4}$, Kun Yan Zhu ${ }^{5}$, Molly Duman-Scheel ${ }^{2,3,4}$ \\ ${ }^{1}$ Division of Biology, Kansas State University \\ ${ }^{2}$ Department of Medical and Molecular Genetics, Indiana University School of Medicine \\ ${ }^{3}$ Eck Institute for Global Health, University of Notre Dame \\ ${ }^{4}$ Department of Biological Sciences, University of Notre Dame \\ ${ }^{5}$ Department of Entomology, Kansas State University \\ * These authors contributed equally
}

Correspondence to: Molly Duman-Scheel at Molly.A.Scheel.2@nd.edu

URL: http://www.jove.com/video/52523

DOI: doi: $10.3791 / 52523$

Keywords: Molecular Biology, Issue 97, vector biology, RNA interference, Anopheles gambiae, Aedes aegypti, dsRNA, siRNA, knockdown, ingestion, mosquito, larvae, development, disease

Date Published: 3/25/2015

Citation: Zhang, X., Mysore, K., Flannery, E., Michel, K., Severson, D.W., Zhu, K.Y., Duman-Scheel, M. Chitosan/Interfering RNA Nanoparticle Mediated Gene Silencing in Disease Vector Mosquito Larvae. J. Vis. Exp. (97), e52523, doi:10.3791/52523 (2015).

\section{Abstract}

Vector mosquitoes inflict more human suffering than any other organism—and kill more than one million people each year. The mosquito genome projects facilitated research in new facets of mosquito biology, including functional genetic studies in the primary African malaria vector Anopheles gambiae and the dengue and yellow fever vector Aedes aegypti. RNA interference- (RNAi-) mediated gene silencing has been used to target genes of interest in both of these disease vector mosquito species. Here, we describe a procedure for preparation of chitosan/ interfering RNA nanoparticles that are combined with food and ingested by larvae. This technically straightforward, high-throughput, and relatively inexpensive methodology, which is compatible with long double stranded RNA (dsRNA) or small interfering RNA (siRNA) molecules, has been used for the successful knockdown of a number of different genes in $A$. gambiae and $A$. aegypti larvae. Following larval feedings, knockdown, which is verified through qRT-PCR or in situ hybridization, can persist at least through the late pupal stage. This methodology may be applicable to a wide variety of mosquito and other insect species, including agricultural pests, as well as other non-model organisms. In addition to its utility in the research laboratory, in the future, chitosan, an inexpensive, non-toxic and biodegradable polymer, could potentially be utilized in the field.

\section{Video Link}

The video component of this article can be found at http://www.jove.com/video/52523/

\section{Introduction}

Blood feeding vector mosquitoes of the Anopheline and Aedine genera transmit disease-causing agents responsible for several of the worst scourges of humankind. An estimated 3.4 billion people are at risk for contracting malaria, which is responsible for over one-half million deaths annually worldwide. Malaria results from infection by Plasmodiumsp. parasites, which are transmitted to people through the bites of infected mosquitoes of the Anopheles genus, including the principal African vector Anopheles gambiae (http://www.who.int/topics/malaria/en/, 2014) ${ }^{1}$. Aedes aegypti is the primary mosquito vector for dengue virus, which causes dengue fever, a nonspecific febrile illness that is the most widespread and significant arboviral disease in the world. Dengue virus is presently a threat to $>2.5$ billion people in the tropics, with a yearly incidence of approximately 50 million cases resulting in $\sim 24,000$ deaths annually (http://www.cdc.gov/dengue/, 2014) ${ }^{2}$. Despite the devastating global impact of mosquito-borne illnesses on human health, effective means of preventing and treating these diseases are lacking. Mosquito control is presently the best method of disease prevention.

The potential for controlling arthropod-borne diseases by the genetic manipulation of vector insects has been recognized for over four decades ${ }^{3}$. Transgenic strains of $A$. aegypti engineered to have a repressible female-specific flightless phenotype have recently made the potential for using transgenic vector control strategies a reality ${ }^{4-6}$. These advancements have challenged researchers to identify novel genetic targets for vector control and additional means of manipulating gene function in vector mosquitoes. Alteration of gene expression during development, as was the case in female-flightless mosquitoes ${ }^{4}$, may promote the elucidation of novel vector control strategies. However, largely due to technical challenges, the functions of very few genes have been characterized during development of $A$. gambiae, $A$. aegypti, or other mosquito species.

Since its discovery in C. elegans ${ }^{7}$, RNA interference (RNAi), which is conserved in animals, plants, and microorganisms, has been extensively used for functional genetic studies in a wide variety of organisms, including insects ${ }^{8,9}$. The RNAi pathway is initiated by Dicer, which cleaves long dsRNA into short 20-25 nucleotide-long siRNAs that function as sequence-specific interfering RNA. siRNAs silence genes that are complementary in sequence by promoting transcript turnover, cleavage, and disruption of translation ${ }^{9}$. Long dsRNA molecules (typically $300-600$ 
bp) or custom siRNAs targeting a particular sequence can be used in the research laboratory for silencing any gene of interest. By managing when interfering RNA is delivered, researchers can control the time at which gene silencing initiates. This advantage is useful as it can be used to overcome challenges such as developmental lethality or sterility, which can hinder the production and maintenance of strains bearing heritable mutations, an expensive and labor-intensive process that is not yet available in all insect species. Although the degree of gene silencing by RNAi can vary from gene to gene, tissue to tissue, and animal to animal, RNAi is widely used for functional analysis of genes in mosquitoes and other insects $^{8,9}$

Three interfering RNA delivery strategies have been used in mosquitoes: microinjection, soaking/topical application, and ingestion. For a detailed history and comparison of the use of these three techniques in insects, please refer to Yu et $a^{8}{ }^{8}$. We have successfully used microinjection ${ }^{10}$ as a means of delivering siRNAs to target developmental genes in $A$. aegypti embryos, larvae, and pupae ${ }^{11-14}$. However, this labor-intensive delivery strategy requires both a microinjection setup and a skilled hand. Moreover, microinjection is stressful to the organism, a confounding factor, particularly when behavioral phenotypes will be assessed. Finally, microinjection delivery cannot be extended to the field for vector control. As an alternative, soaking the organism in interfering RNA solution has also become a popular means of inducing gene silencing, as it is convenient and requires little equipment or labor. Soaking has primarily been applied in insect cell line studies ${ }^{8}$, but in a recent study, knockdown was achieved in $A$. aegypti larvae immersed in a solution of dsRNA ${ }^{15}$, which the animals appeared to be ingesting. However, for studies involving analysis of multiple experimental groups or phenotypes, soaking is rather costly. Lopez-Martinez et al. ${ }^{16}$ described rehydration driven RNAi, a novel approach for interfering RNA delivery that involves dehydration in saline solution and rehydration with a single drop of water containing interfering RNA. This approach does cut the costs associated with whole animal immersion, but is more expensive than microinjection and may be limited in its application to species that can tolerate high osmotic pressures. Moreover, it is difficult to envision how immersion or dehydration/ rehydration immersion methodology could be adapted for vector control in the field. For these reasons, for post-embryonic studies, delivery of interfering RNA with ingested food is a viable alternative strategy.

Although ingestion-based strategies do not work in all insect species, perhaps most notably Drosophila melanogaster, oral delivery of interfering RNA mixed with food has promoted gene silencing in a variety of insects ${ }^{8,17}$, including $A$. aegypti adults ${ }^{18}$. We described chitosan nanoparticlemediated RNAi in A. gambiae larvae ${ }^{19}$ and have successfully applied this approach for reduction of gene expression in $A$. aegypti larvae ${ }^{20,21}$. Here, methodology for this RNAi procedure, which involves entrapping of interfering RNA by the polymer chitosan, is detailed. Chitosan/ interfering RNA nanoparticles are formed by self-assembly of polycations with interfering RNA through the electrostatic forces between positive charges of the amino groups in chitosan and negative charges carried by the phosphate groups on the backbone of interfering RNA ${ }^{19}$. The procedure described is compatible with both long dsRNA molecules (hereafter referred to as dsRNA) or double stranded siRNA (hereafter referred to as siRNA). Following synthesis, chitosan/interfering RNA nanoparticles are mixed with larval food and delivered to larvae through oral ingestion. This methodology is relatively inexpensive, requires little equipment and labor ${ }^{19}$, and facilitates high-throughput analysis of multiple phenotypes, including analysis of behaviors ${ }^{20,21}$. This methodology, which can be adapted for gene silencing studies in other insects, including other disease vectors and insect agricultural pests, could potentially be used for gene silencing in a variety of other animal species. Moreover, chitosan, an inexpensive, non-toxic and biodegradable polymer ${ }^{22}$, could potentially be utilized in the field for species-specific mosquito control.

\section{Mosquito Species and Rearing}

1. Maintain A. gambiae G3 and A. aegypti Liverpool IB12 strains (used in the representative studies below) or other strains of interest according to standard lab practice or as described previously ${ }^{23,24}$.

\section{2. dsRNA and siRNA Design and Production}

1. Design primers to construct long dsRNA templates specific to the gene of interest. Use the E-RNAi tool ${ }^{25}$. Produce dsRNA according to the method of choice or as previously described ${ }^{19}$.

1. For a negative control, synthesize dsRNA ${ }^{19}$ corresponding to the sequence of green fluorescent protein (GFP), $\beta$-galactosidase ( $\beta$-gal), or some other gene not expressed in mosquitoes. If so desired, dsRNA ${ }^{19}$ utilized to generate the representative results summarized below can be used as a positive control. Store dsRNA dissolved in RNAse-free water at $-80{ }^{\circ} \mathrm{C}$ until needed.

2. Alternatively, design gene-specific siRNA according to standard lab practice or as described previously ${ }^{10}$. Scramble the sequence of a knockdown siRNA to design negative control siRNA that does not correspond to any mosquito gene. Purchase the custom siRNAs, which are available through a number of reputable vendors.

1. If so desired, siRNAs ${ }^{20,21}$ utilized to obtain the representative results summarized below can be used as positive controls. Store siRNA dissolved in RNAse-free water at $-80{ }^{\circ} \mathrm{C}$ until needed.

\section{Preparing Chitosan/interfering RNA Nanoparticles}

1. Gather pre-made RT $100 \mathrm{mM}$ sodium sulfate $\left(100 \mathrm{mM} \mathrm{Na}_{2} \mathrm{SO}_{4}\right.$ in deionized $\left.\mathrm{H}_{2} \mathrm{O}\right)$ and $0.1 \mathrm{M}$ sodium acetate $\left(0.1 \mathrm{M} \mathrm{NaC}_{2} \mathrm{H}_{3} \mathrm{O}_{2}-0.1 \mathrm{M}\right.$ acetic acid, $\mathrm{pH} 4.5$ in deionized $\mathrm{H}_{2} \mathrm{O}$ ) buffers.

2. Dissolve chitosan ( $\geq 75 \%$ deacetylated) in $0.1 \mathrm{M}$ sodium acetate buffer to make $0.02 \%(\mathrm{w} / \mathrm{v})$ working solution and keep the solution at $\mathrm{RT}$ before use.

3. Dissolve dsRNA or siRNA in $50 \mu \mathrm{l}$ deionized $\mathrm{H}_{2} \mathrm{O}$ and add it to the $100 \mathrm{mM}$ sodium sulfate buffer to make a $100 \mu \mathrm{l}$ solution of $32 \mu \mathrm{g}$ of dsRNA/siRNA per $100 \mu$ of $50 \mathrm{mM}$ sodium sulfate.

4. Add $100 \mu \mathrm{l}$ of chitosan solution to the dsRNA/siRNA solution and then heat the mixture in a water bath at $55^{\circ} \mathrm{C}$ for $1 \mathrm{~min}$. Set up a control by adding $100 \mu \mathrm{l}$ of $50 \mathrm{mM}$ sodium sulfate to $100 \mu \mathrm{l}$ of chitosan solution and follow the same procedure.

5. Mix the solutions immediately for $30 \mathrm{sec}$ by high-speed vortexing at RT to facilitate the formation of nanoparticles. 
6. Centrifuge the mixture at $13,000 \times \mathrm{g}$ for $10 \mathrm{~min}$ at $\mathrm{RT}$, after which time a pellet should be visible. Transfer the supernatant to a new $1.5 \mathrm{ml}$ tube. Air-dry the pellet for $\approx 10 \mathrm{~min}$ at RT before using it to prepare mosquito food.

7. Measure the concentration of dsRNA/siRNA in the supernatant by using the supernatant from the control (see 3.5) as a blank to calculate the total amount of dsRNA/siRNA that remained in the supernatant. Use the difference between starting amounts of dsRNA/siRNA and amounts remaining in the supernatants to calculate the percentage of dsRNA/siRNA entrapped in the nanoparticles. This loading efficiency is normally over $90 \%$.

8. Repeat the same procedure if more nanoparticles are needed. Use the dried nanoparticles immediately. The impact of cold storage of particles prior to use has not been evaluated.

\section{Preparing Mosquito Food containing Chitosan/interfering RNA Nanoparticles}

1. Prepare $1 \mathrm{ml}$ of a $2 \%$ agarose solution $(\mathrm{w} / \mathrm{v})$ in deionized water, melt the agarose, and keep melted agarose solution in a $55{ }^{\circ} \mathrm{C}$ water bath before use.

2. Mix fish food flakes ( $47 \%$ crude protein, min. crude fat $10 \%$, max. crude fiber $3 \%$ ) and dry yeast at a ratio of $2: 1$ (w/w). Grind the mixture to small particles with a mortar and pestle (passable through No. 50 USA standard test sieve). Use the ground food, which should be brownish in color, either immediately or store it in a sealed container at $4{ }^{\circ} \mathrm{C}$ for several weeks.

3. In a $1.5 \mathrm{ml}$ tube, mix $6 \mathrm{mg}$ of ground food with the dried nanoparticles from Section 3.7 with a toothpick.

4. Add $30 \mu \mathrm{l}$ of $2 \%$ pre-melted agarose gel solution to the food-nanoparticle mixture; stir immediately and thoroughly by using a toothpick or pipet tip.

5. Use the gel containing food and nanoparticles to feed the mosquito larvae immediately. Alternatively, once the gel is completely solidified at $\mathrm{RT}$, store the gel at $4{ }^{\circ} \mathrm{C}$ and use the next day, or at $-80^{\circ} \mathrm{C}$ for later use.

\section{Feeding Mosquito Larvae with Food Containing Chitosan/interfering RNA Nanoparticles}

1. Feeding A. gambiae mosquito larvae:

1. Remove a single gel pellet from the $1.5 \mathrm{ml}$ tube using a toothpick and cut it into 6 equal slices using a clean razor blade or tooth pick.

2. Transfer 20 third instar larvae to a $500 \mathrm{ml}$ Petri dish containing $100 \mathrm{ml}$ of deionized water.

3. Feed the mosquito larvae by adding one slice of the gel pellet (finely chopped into smaller pieces) per Petri dish once a day for four days. Be sure to observe larvae feeding on the pellet, which should be significantly reduced in size or completely absent by the next day. After time, mosquitoes will develop into late fourth instar larvae.

4. Record any visible phenotypic changes during the experiment. Examine the transcript levels and other phenotypic changes as discussed in section 6 at the end of the four day period.

2. Feeding $A$. aegypti mosquito larvae:

1. Cut the gel pellet into 6 equal slices using a clean razor blade or toothpick.

2. Place 50 age-synchronized $24 \mathrm{hr}$ after egg hatching first instar larvae into a petri dish in $\approx 40 \mathrm{ml}$ deionized water.

3. Feed larvae one slice per petri dish for $4 \mathrm{hr} /$ day, then transfer larvae back to the regular larval diet of $2: 1 \mathrm{ground}$ fish food flakes and dry yeast for the rest of the day. Repeat procedure daily throughout the four larval instars.

4. Examine the transcript levels and other phenotypic changes as discussed in section 6 at the desired developmental time points.

\section{Confirmation of Gene Knockdown}

1. Relative transcript quantification by qRT-PCR in $A$. aegypti and A. gambiae larvae

1. Perform and analyze qRT-PCR assays with three biological replicates on at least 10 pooled control vs. experimental larvae as described ${ }^{11,19,20}$, or according to standard lab procedure. Representative results are included below.

2. For analysis of a particular tissue type/body part (i.e., brain or antenna), perform qRT-PCR as described in 6.1.1 following dissection to recover the tissue of interest (for example, see Mysore et al. ${ }^{21}$ ).

2. Confirmation of knockdown by in situ hybridization

1. Synthesize digoxygenin-labeled antisense and sense control riboprobes according to standard lab practice or as described ${ }^{26}$

2. Execute in situ hybridization per the Haugen et al. ${ }^{27}$ protocol for confirmation of knockdown as described previously ${ }^{11,20}$ and discussed in the representative results section below.

3. Confirmation of knockdown by immunohistochemistry

1. If antibodies against the protein product of the gene targeted are available, perform immunohistochemistry as previously described ${ }^{28}$, which facilitates identification of individuals with the greatest levels of knockdown for phenotype analysis ${ }^{20}$ as exemplified in the representative results section below.

Representative Results

\section{A. gambiae:}

Chitosan/dsRNA nanoparticles are formed due to the electrostatic interaction between amino groups of chitosan and the phosphate groups of dsRNA. The efficiency of dsRNA incorporation into nanoparticles is usually above $90 \%$ as measured by depletion of dsRNA from the solution. Atomic force microscopy images show that chitosan-dsRNA particle size averages $140 \mathrm{~nm}$ in diameter, ranging from 100-200 nm (Figure 1). 


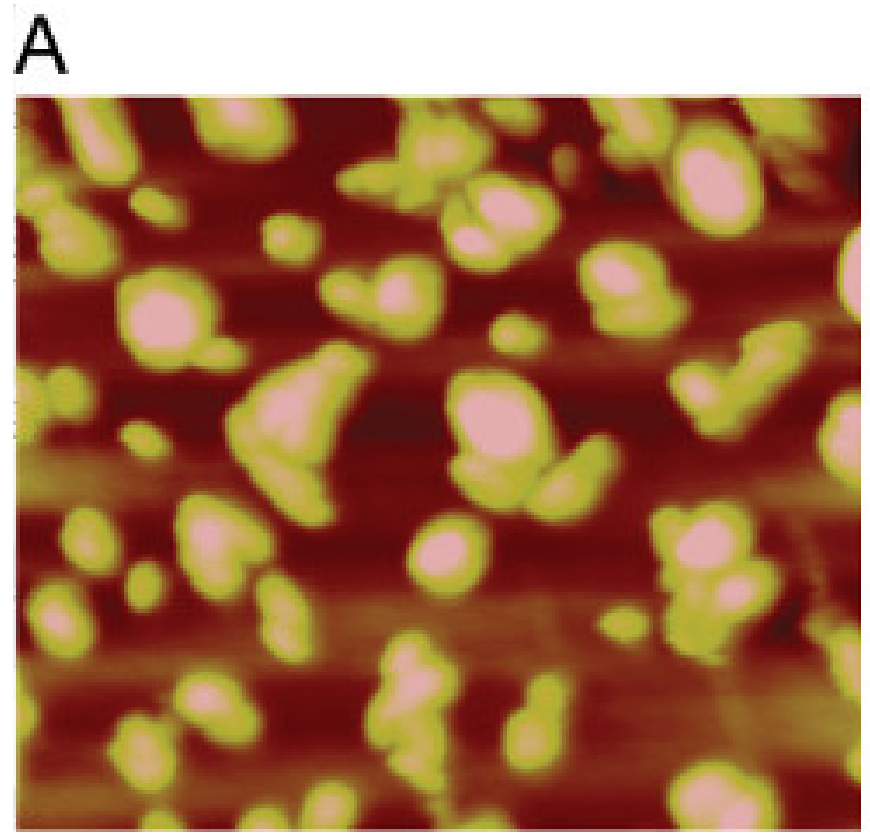

\section{B}

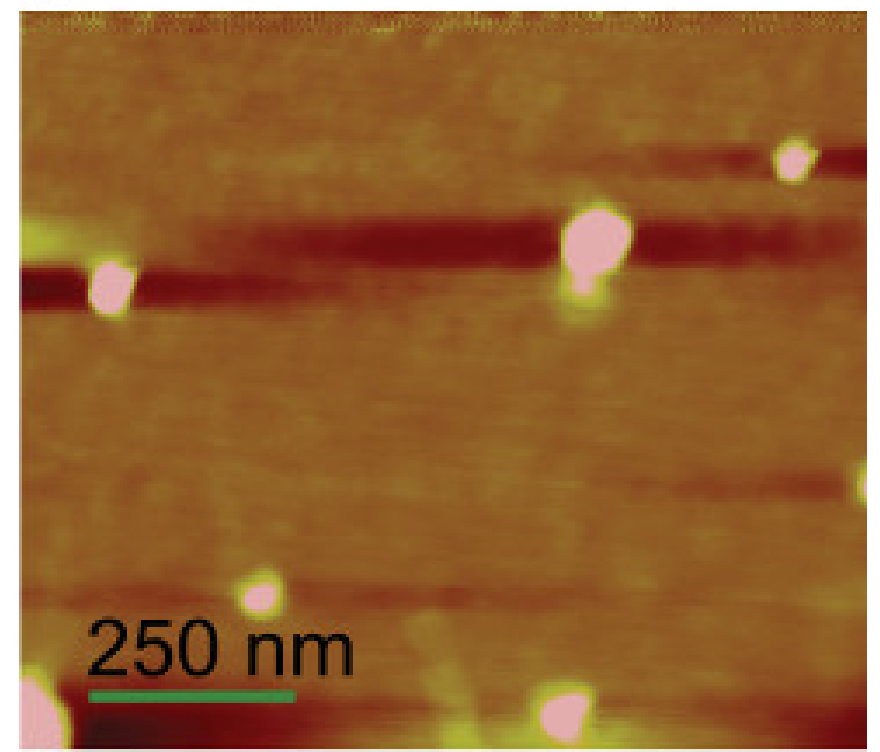

Figure 1. Nanoparticle formation by chitosan and interfering RNA. Atomic force microscopy image of chitosan/dsRNA nanoparticles (A) or chitosan solution without the addition of dsRNA (B). The size of the images was $1.0 \mu \mathrm{m} \times 1.0 \mu \mathrm{m}$. Scale bar $=250 \mathrm{~nm}$. Please click here to view a larger version of this figure.

Feeding of these particles which are combined with standard larval food and incorporated into agarose supports normal larval development. Importantly, specific knockdown of ectoderm-derived AgCHS1 transcripts as well as midgut-specific AgCHS2 (Figure 2) is possible ${ }^{19}$, demonstrating that dsRNA taken up through ingestion of nanoparticles initiates RNAi beyond the midgut. Knockdown efficiencies $\approx 40-60 \%$ were observed (e.g., Figure 2) and vary between transcripts. 


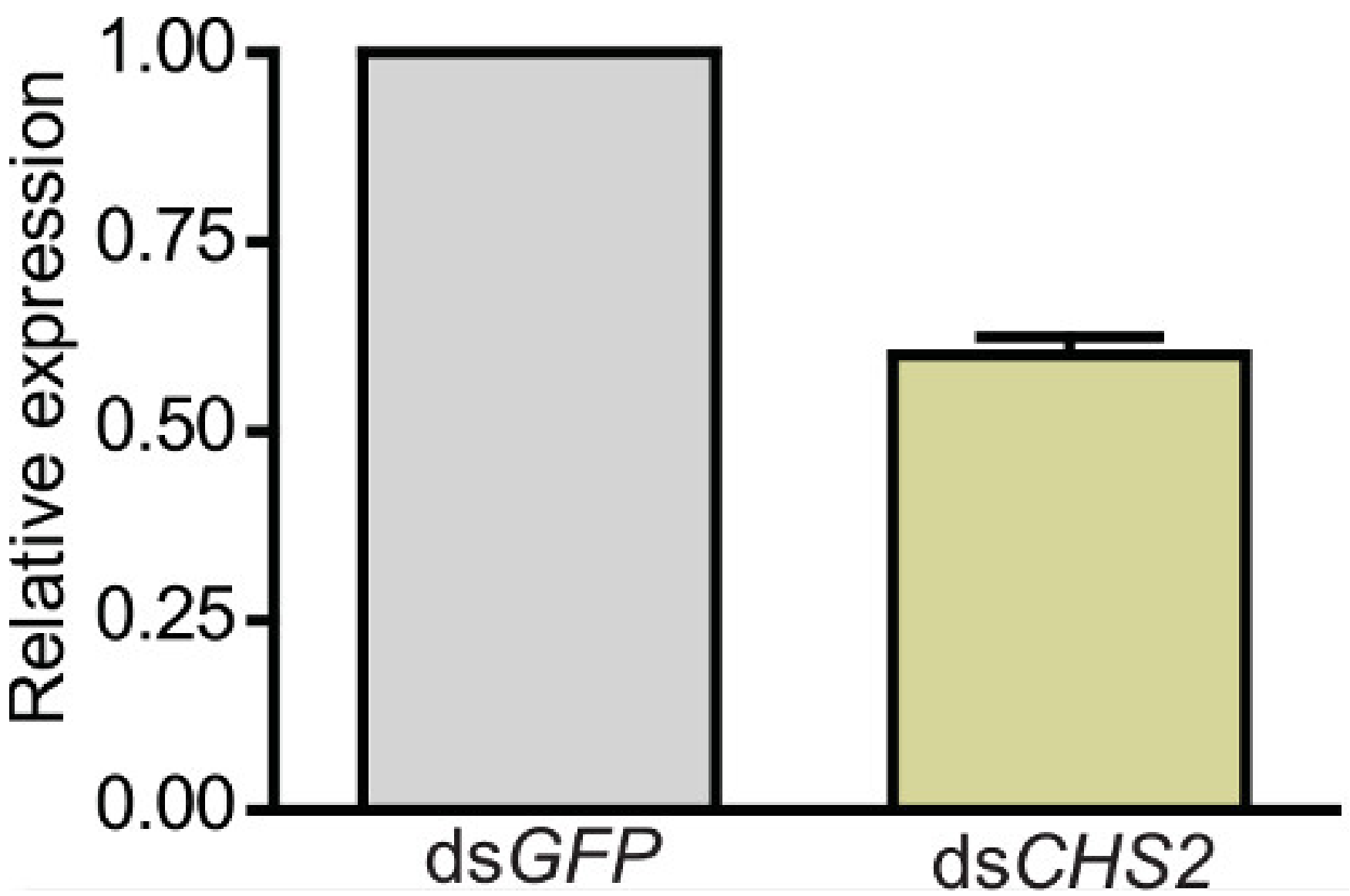

Figure 2.Transcription levels of chitin synthase 2 (AgCHS2) after dsRNA ingestion in A. gambiae larvae. Relative mRNA levels of $A g C H S 2$ in larvae fed on nanoparticles with ds $A g C H S 2$ or control dsGFP. Data are presented as mean \pm SD from three independent biological replicates. Different letters on the bars indicate significant differences based on paired tests $(P=0.003)$.

Knockdown of $A g C H S 1$ significantly reduced the total chitin content of fourth instar larvae and increased the susceptibility to the chitin synthesis inhibitor insecticide, diflubenzuron ${ }^{19}$, Knockdown of $A g C H S 2$ significantly increased the effect of calcofluor white and dithiothreitol which disrupted the peritrophic matrix (PM) in fourth instar larvae (Figure 3) resulting in increased mortality ${ }^{17}$. 


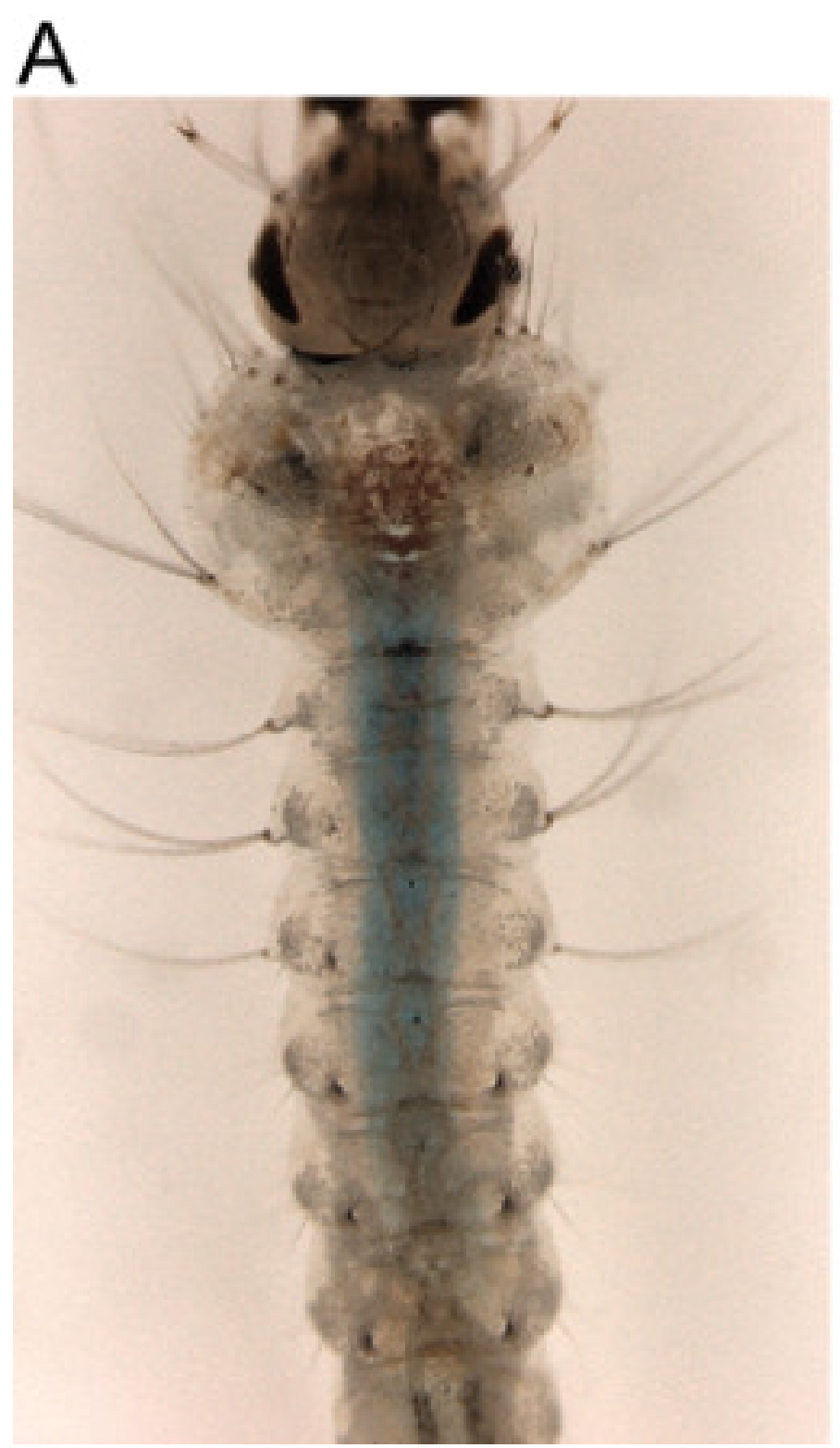

\section{B}

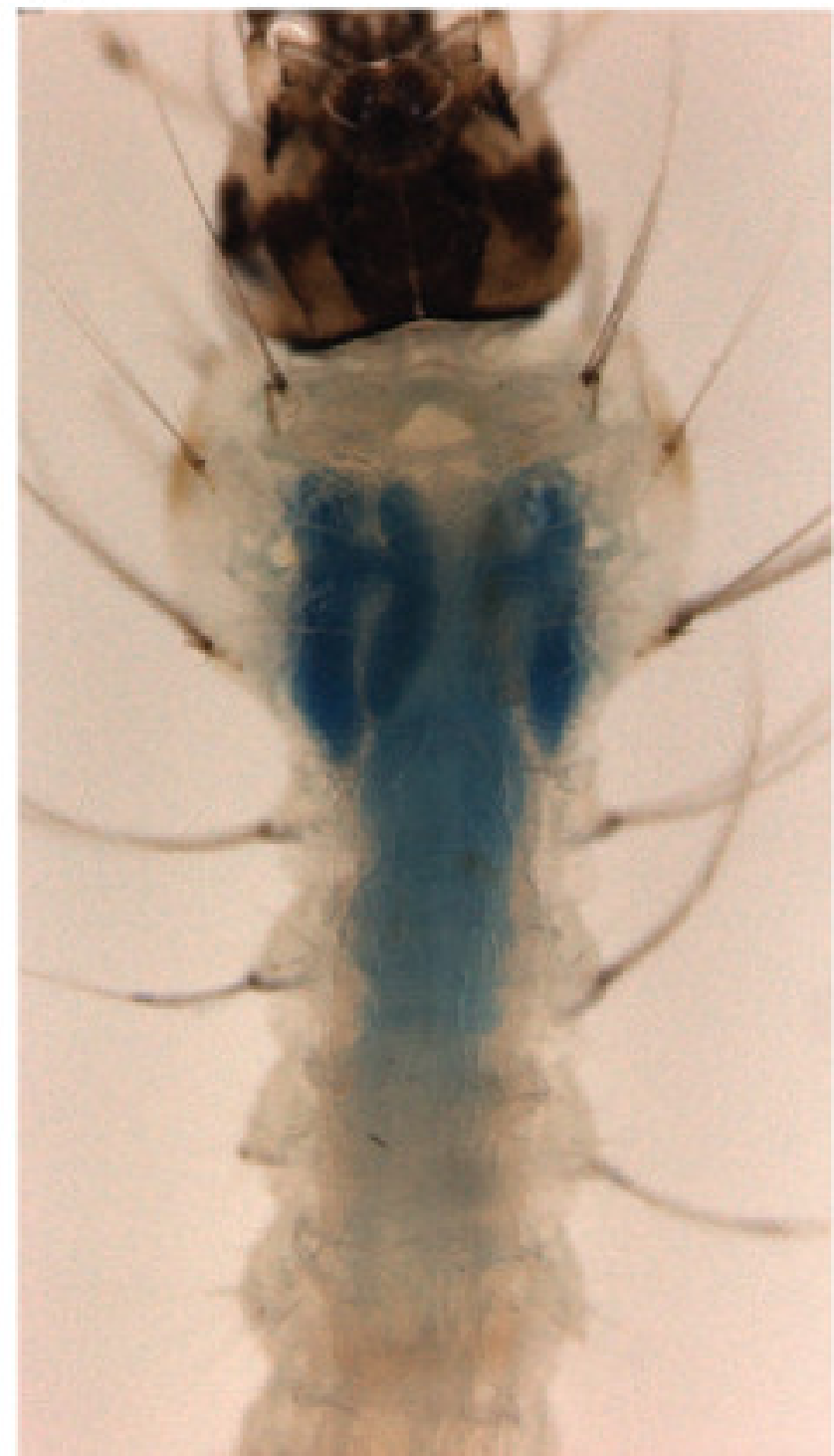

Figure 3. Impact of chitosan/ds $A$ gCHS2 ingested nanoparticles on $A$. gambiae peritrophic matrix (PM) permeability. Calcofluor white or DTT treatment disrupts the PM of $A$. gambiae larvae which is further exuberated by $A g C H S 2$ knockdown through ingestion of the chitosan/ dsRNA nanoparticles ${ }^{19}$. (A) Mosquito with intact PM. (B) Mosquito with disrupted PM, dextran blue leaking into the gastric ceacae.

\section{A. aegypti:}

Chitosan/siRNA nanoparticles were used to target the axon guidance gene semaphorin-1a (sema1a) during A. aegypti larval development ${ }^{20}$. Knockdown of sema1a was confirmed through in situ hybridization, which detected reduced levels of sema1a in $\approx 60 \%$ of the larval brains assessed and failed to detect sema1a expression in $\approx 40 \%$ of knockdown animal brains (Figure4C vs. B; yellow arrowhead indicates the antennal lobe). qRT-PCR assays performed on pooled whole animals revealed that this technique resulted in $32 \pm 10 \%$ reduction in sema1a transcripts as compared to control-nanoparticle fed animals (Figure $4 \mathrm{~A} ; P<0.01$ based on paired test, $\mathrm{N}=5$, where $\mathrm{N}$ is the number of biological replicates). Knockdown in the brain persisted through at least $24 \mathrm{~h}$ of pupal development, as evidenced by loss of anti-Sema1a antibody expression (Figure 4E1 vs. D1), which was used to identify animals with significant knockdown during larval and pupal olfactory phenotype analyses. Larval and pupal antennal lobe defects (quantified in Table 1) were detected in sema1a knockdown larvae and pupae (Figure 4E2, E3 vs. D2, D3). Loss of Sema1a expression resulted in glomeruli that are deformed (Figure 4E2 vs. D2, visualized with mAbnc82; overlays of both signals are shown in Figure4D3, E3). Comparable phenotypes were generated with two separate sema1a knockdown siRNAs, which helped to ensure that the defects observed were not the result of off-site targeting by either siRNA. The highest levels of knockdown were generated when the siRNAs were combined, a strategy that can be used to increase knockdown efficiency (see Mysore et al..$^{20}$ for more details). 

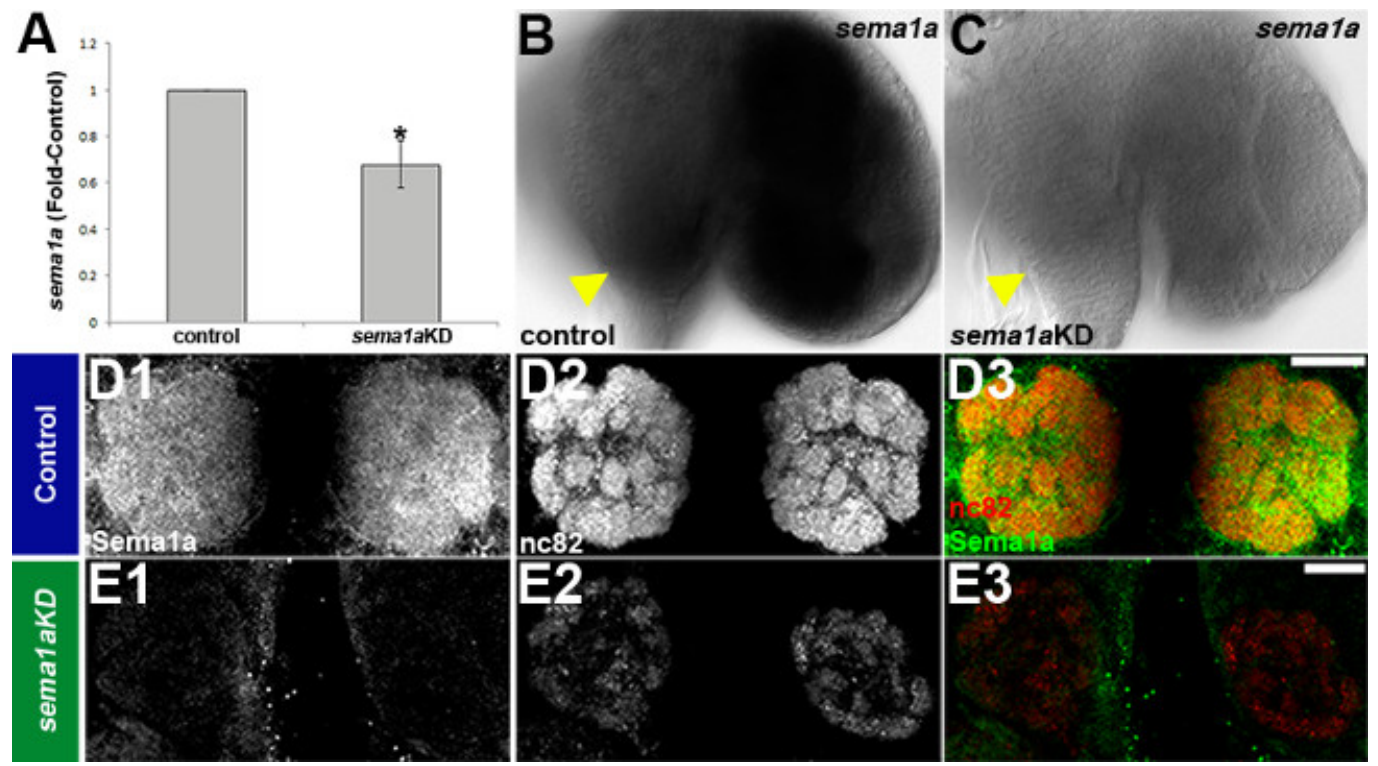

Figure 4. Chitosan/siRNA induced knockdown of sema1a in the developing olfactory system of $\boldsymbol{A}$. aegypti. qRT-PCR (A) and in situ hybridization (B, C) assays confirmed knockdown of sema1a in larvae fed a mixture of sema1a targeting siRNA ${ }^{890}$ and siRNA ${ }^{1198}$ chitosan/ interfering RNA nanoparticles vs. control nanoparticles. Loss of Sema1a expression resulted in glomeruli that are deformed (E1-3 vs. D1-3). See text for details. Dorsal is up in all panels. Scale bar $=25 \mu \mathrm{m}$. This figure originally appeared in Mysore et al ${ }^{20}$. Please click here to view a larger version of this figure.

\begin{tabular}{|c|c|c|c|c|c|c|}
\hline & Larvae & & & Pupae & & \\
\hline SIRNA & $\mathrm{n}$ & Wildtype & AL defects & $n$ & Wildtype & AL defects \\
\hline Control & 69 & $69(100 \%)$ & $0(0 \%)$ & 68 & $68(100 \%)$ & $0(0 \%)$ \\
\hline sema1a KD & 77 & $42(54.5 \%)$ & $35(44 \%)^{*}$ & 75 & $51(68 \%)$ & $24(32 \%)^{*}$ \\
\hline
\end{tabular}

Table 1. Quantification of antennal lobe defects following sema1a knockdown. A compiled summary of results obtained for control siRNA vs. sema1a knockdown (KD) siRNA ${ }^{890}+$ siRNA ${ }^{198}$ chitosan nanoparticle-fed animals from a total of eight replicate experiments performed in both larvae (left) and pupae (right) is shown. The total number of individuals assessed ( $n$ ) and numbers/percentages of animals displaying wild type morphology vs. antennal lobe (AL) defects (olfactory receptor neuron targeting defects, defective neuropil and glomeruli formation) are indicated. Knockdown was verified immunohistochemically with anti-Sema1a antibody staining in a subset of animals (15 larvae and 10 pupae) that displayed the most severe defects (denoted with *). All knockdown animals evaluated in this manner were found to have reduced levels of Sema1a, while Sema1a levels in control-fed animals were not noticeably altered. This table originally appeared in Mysore et al ${ }^{20}$.

In a second investigation ${ }^{21}$, chitosan/siRNA nanoparticles were used to target the transcription factor Single-minded (Sim) during development of the olfactory system. qRT-PCR assays with pooled brains dissected from whole animals indicated that sim knockdown brains had on average a $47 \%$ reduction in sim transcripts in comparison to control nanoparticle-fed animals $(P=0.005$ based on paired t test). Comparable assays with antennae revealed on average a $77 \%$ reduction in sim levels with respect to control-fed animals $(P=0.002$ based on paired t test). Despite some variability in the levels of knockdown between tissues and animals, sim transcripts were not detected through in situ hybridization in $\approx 50 \%$ of the knockdown animal brains/antennae following treatment with either of two different knockdown siRNAs (Figure 5B2, B3, C2, C3 vs. B1, C1, Table 2). In yeast behavioral assays during which individuals that were attracted to yeast were awarded a score of 1 , while animals that were not attracted were given a score of 0 , average scores for $\operatorname{sim}^{430}$ or $\operatorname{sim}^{718}$ knockdown animals were significantly lower than that of control-fed animals in two replicate experiments (Figure 5A; $P<0.001$ based on paired t test). Defective odor-tracking behavior (Figure 5A) correlated with reduced levels of sim in the antennae (Figure 5B2, B3 vs. B1) and brains (Figure 5C2, C3 vs. C1, yellow dots mark the perimeter of the antennal lobe; see Table 2 for quantification of results). Multiple defects were identified in the antennae and antennal lobes of sim knockdown animals (see Mysore et al. ${ }^{21}$ for details). For example, sim knockdown larvae and pupae lacked expression of orco, the obligate co-receptor for all odorant receptors in olfactory receptor neurons (Figure 6). Reduced orco transcript levels were detected in individuals fed with $\operatorname{sim}^{430}$ (Figure 6A3, B3) or $\operatorname{sim}^{718}$ (Figure 6A4, B4) knockdown (KD) chitosan/siRNA nanoparticles (compare to wild type animals in Figure 6A1, B1 and control chitosan/ siRNA nanoparticle-fed animals in Figure 6A2, B2). The loss of orco expression phenotype observed in L4 larvae (Figure 6A1-A4) persisted through at least $24 \mathrm{~h}$ after pupal formation (Figure 6B1-B4). 


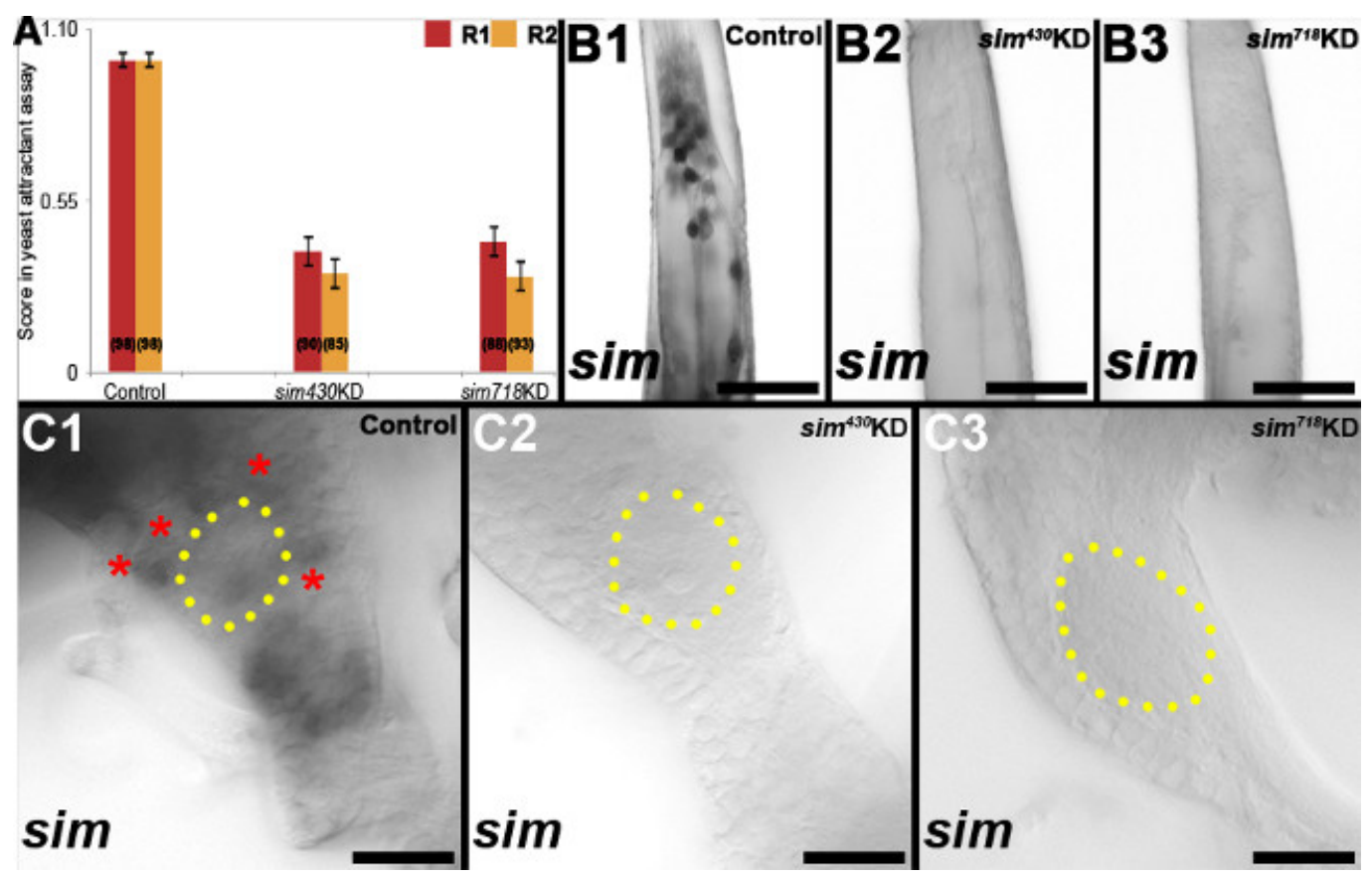

Figure 5. sim knockdown larvae show defective odor-attractant behavior. In situ hybridization revealed reduced levels of sim RNA in the antennae (B2, B3) and brains (C2, C3) of $\operatorname{sim}^{430}$ and $\operatorname{sim}^{718}$ knockdown animals (compare to control-fed in B1, C1), which failed to respond to yeast odorant attractant (A). See text for details. The proximal ends of antennae are oriented upwards in B1-B3. Dorsal is oriented upward in C1C3. Scale bar $=25 \mu \mathrm{m}$. This figure originally appeared in Mysore et $a^{21}$. Please click here to view a larger version of this figure.

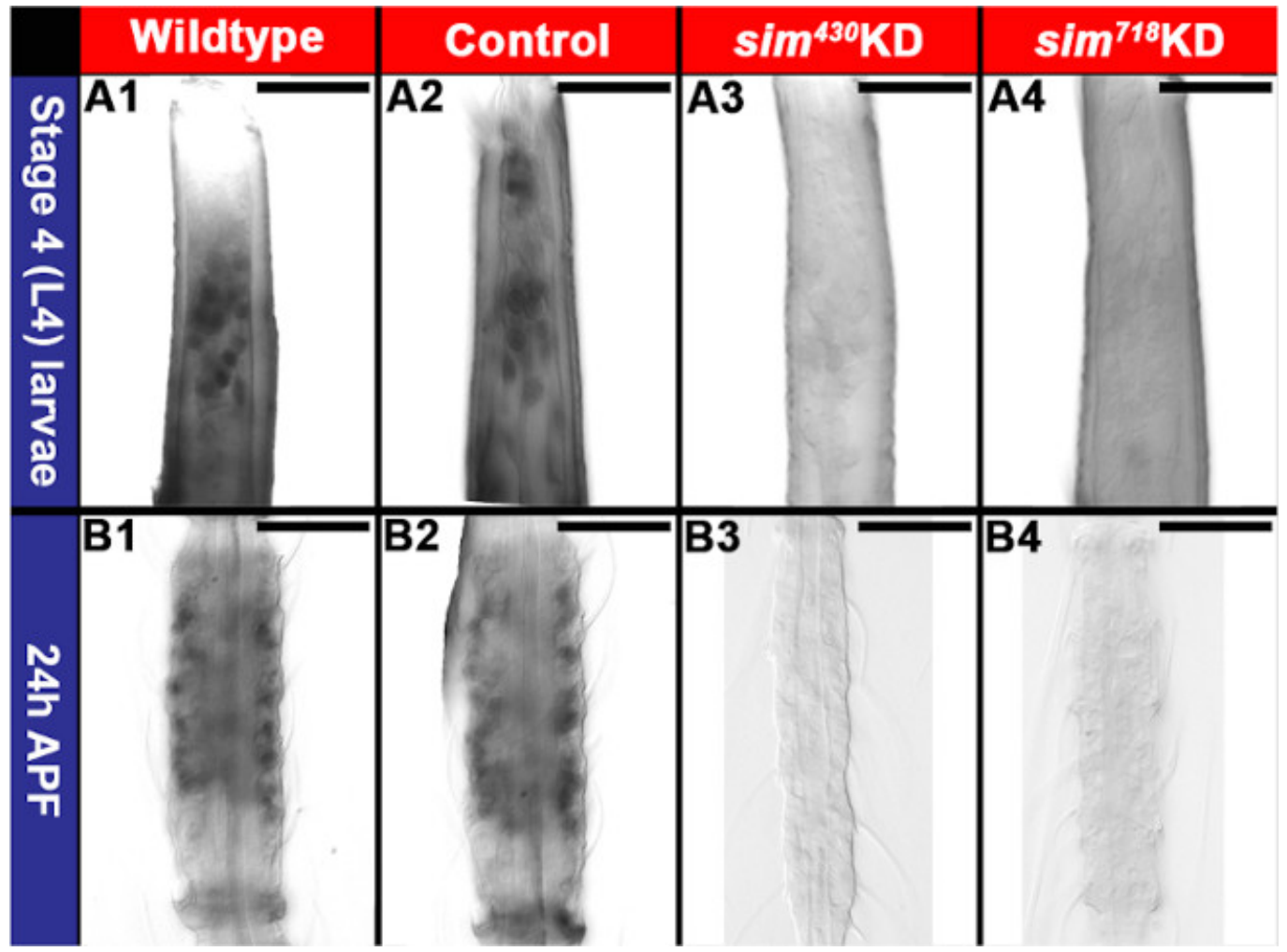

Figure 6. Loss of orco expression in the developing $A$. aegypti antenna following knockdown of sim. Reduced levels of orco transcript were detected in individuals fed with $\operatorname{sim}^{430}(\mathbf{A} 3, \mathbf{B} 3)$ or $\operatorname{sim}^{718}$ (A4, B4) knockdown (KD) chitosan/siRNA nanoparticles (compare to wild type animals in A1, B1 and control chitosan/siRNA nanoparticle-fed animals in A2, B2). See text for details. Scale bar $=25 \mu \mathrm{m}$. This figure was compiled from images published in Mysore et $\mathrm{al}^{21}$. Please click here to view a larger version of this figure. 


\begin{tabular}{|l|l|l|l|l|l|l|l|l|l|}
\hline & \multicolumn{4}{|c|}{ Attracted } & \multicolumn{3}{l|}{ Not Attracted } \\
\hline siRNA & $\mathbf{n}$ & \# Animals & Normal & Moderate & Null & \# Animals & Normal & Moderate & Null \\
\hline Control & 195 & $196(100 \%)$ & $196(100 \%)$ & 0 & 0 & 0 & 0 & 0 \\
\hline $\operatorname{sim}^{430}$ KD & 176 & $63(35 \%)$ & $48(76 \%)$ & $15(24 \%)$ & 0 & $113(64 \%)$ & $20(18 \%)$ & $12(11 \%)$ & $81(71 \%)$ \\
\hline $\operatorname{sim}^{718}$ KD & 177 & $66(37 \%)$ & $44(66 \%)$ & $22(34 \%)$ & 0 & $111(63 \%)$ & $20(18 \%)$ & $9(8 \%)$ & $82(74 \%)$ \\
\hline
\end{tabular}

Table 2. Levels of sim correlate with larval performance in a yeast behavioral assay. A compiled summary of results obtained in four yeast odorant attractant replicate experiments is shown. The total number of animals $(n)$ indicates the number of individual larvae that were tested in these behavioral assays. The number of individual larvae (\# Animals) that were attracted (left; animals that touched the yeast pellet and were awarded a score of 1) or not attracted (right; animals that did not touch the yeast pellet and received a score of 0 ) under each condition (Control, $\operatorname{sim}^{430} \mathrm{KD}$, or $\operatorname{sim}^{718} \mathrm{KD}$ chitosan/siRNA nanoparticle-fed) are shown, and the percentages of total animals are included following the raw numbers. Knockdown of sim was assessed through in situ hybridization in the brains and antennae of animals attracted (left) or not attracted (right) to the yeast. The raw number/percentage of individuals with Normal (comparable to wildtype sim transcript levels), Null (no observable sim transcript), or Moderate (reduced but not wild type) sim levels are indicated. Loss of sim correlated well with a lack of attraction to the yeast. This table originally appeared in Mysore et $a^{21}$.

\section{Discussion}

The chitosan/interfering RNA nanoparticle methodology described herein has been used to effectively target genes during larval development in A. gambiae (Figures 2, 3) and $A$. aegypti (Figures 4, 5, 6, Tables 1, 2). Chitosan nanoparticles can be prepared with either long dsRNA or siRNA, both of which have been used successfully in mosquitoes as evidenced by the representative results described herein. Synthesis of dsRNA is less expensive than purchasing siRNA, but is more labor intensive. If chitosan/siRNA is used, phenotypes can be confirmed with multiple siRNAs, allowing for better assurance that the phenotype uncovered is not due to off-site targeting. Also, the commercial production of siRNAs en masse may better facilitate expansion of chitosan/interfering RNA silencing for species-specific mosquito control to the field. This will of course require overcoming obstacles such as cost and delivery.

Although we have enjoyed good success with this protocol, methodologies must be optimized for each gene. For this reason, we recommend testing multiple siRNAs, or perhaps one siRNA and a long dsRNA, at the onset of a new project. Once phenotypes are identified and confirmed with at least two distinct siRNAs corresponding to a gene of interest, it may be useful to combine these siRNAs to increase phenotype penetrance (Figure 4). The timing and also the duration of chitosan/interfering RNA feeding time can be optimized for each study. A. gambiae thrive when fed only with chitosan/interfering RNA food as described herein. However, in the experiments we have performed to date, $A$. aegypti have not fared well without supplemental nutrition, a problem we have overcome by transferring them to a diet of normal food after $4 \mathrm{~h}$ of chitosan/interfering RNA feeding. Experimenting with chitosan/interfering RNA delivery in a different food substrate, which we have not yet attempted, may circumvent this problem. We have found that $4 \mathrm{~h}$ daily feedings over the course of several days result in a good balance between knockdown and adequate nutrition, but modification of the duration of feeding could be pursued further as desired. Also, the larval instar in which chitosan/interfering RNA nanoparticle feeding is initiated/continued can be tailored for each experiment depending on the critical developmental period in which gene function is examined, a major advantage of this protocol.

Verification of knockdown is of course critical, but may also require troubleshooting. For this reason, it may be useful to simultaneously check if phenotypes of interest are being generated as knockdown efficiency is being assessed. For qRT-PCR validation experiments, it is critical that primers and PCR conditions are optimized. Moreover, it is important that animals are developmentally synchronized at the onset of the experiment, as expression of some transcripts can be very dynamic during development and due to circadian rhythms ${ }^{29}$. Furthermore, while this technique clearly generates knockdown beyond the gut, we are only beginning to assess the tissues for which this technique is effective, and this may vary among species. It will therefore be useful to measure knockdown in particular tissues, which can be achieved by dissecting tissues of interest from whole animals for qRT-PCR assays or through in situ hybridization (Figures 4-6) ${ }^{20,21}$ and immunohistochemistry (Figure 4) ${ }^{20}$ assays (see Haugen et al. ${ }^{27}$ and Clemons et al. ${ }^{28}$, respectively, for troubleshooting these protocols). Since knockdown varies from individual to individual, it is helpful to perform double-labeling assays to assess phenotypes in combination with knockdown levels (Figure 4) ${ }^{20}$, which greatly facilitates phenotype characterization. For behavioral studies, knockdown levels in individual animals can be correlated with their behavioral performances (Table 2) ${ }^{20,21}$.

Following chitosan-mediated interfering RNA delivery to $A$. aegypti larvae, reduced gene expression is detected at $24 \mathrm{~h}$ of pupal development (Figures 4, 6) ${ }^{20,21}$. Although levels of gene expression have not yet been assessed in a detailed manner beyond this point of development, reduced levels of gene expression have been detected in both 48 and $72 \mathrm{~h} A$. aegypti pupae (KM, unpublished). It would be useful to pursue more detailed analyses of knockdown levels at multiple stages of $A$. aegypti pupal development and also to assess $A$. gambiae pupae. It would also be interesting to determine whether knockdown persists in adults and to explore nanoparticle-mediated interfering RNA delivery strategies for adult mosquitoes.

Site-specific nuclease gene targeting approaches were recently introduced to $A$. aegypti and $A$. gambiae and are permitting the generation of targeted, heritable mutations in mosquitoes and other non-genetic model organisms ${ }^{30,31}$. Although use of these approaches permits more uniform loss of function phenotype analyses, an advantage over RNAi approaches, screening for mutations of interest is still fairly time consuming and labor-intensive. Furthermore, although RNAi loss of function studies require maintenance of only wild type strains, mutant mosquito strains must be individually reared. The maintenance of recessive lethal or adult sterile mutations is presently challenging given the current lack of marked balancers in mosquitoes. Thus, while site-specific nuclease gene targeting approaches are quickly gaining popularity, chitosan/interfering RNA targeting may be the optimal choice for laboratories not equipped to maintain mosquito strains and in cases for which loss of gene function during development is incompatible with survival of the strain. 
Based on our findings, we anticipate that chitosan/interfering RNA can be used broadly for knockdown of many different genes during development of $A$. gambiae, $A$. aegypti, as well as other insects, and potentially other non-genetic model organisms. Successful gene silencing with chitosan/interfering RNA has been reported in other arthropod species, including Penaeus monodon (shrimp) ${ }^{32}$. A recent study demonstrated that nanoparticles facilitate the uptake of dsRNA and thus enhance the knockdown efficiency as compared with direct feeding of dsRNA in the Asian cornborer ${ }^{33}$, which underscores the potential for using this technology to target agricultural pests. The possible use of siRNA-nanoparticles as therapeutics in humans is attracting a great deal of interest in the medical community. Giljohann et al. ${ }^{34}$ described the synthesis and characterization of polyvalent RNA-gold nanoparticle conjugates, which have a six-fold longer half-life in comparison to free dsRNA and readily enter cells. Davis et al. ${ }^{35}$ described the first human clinical trial involving systemic administration of siRNAs using a nanoparticle delivery system to patients with solid tumors, and the possibility of using chitosan/siRNA therapeutics was recently reviewed ${ }^{36}$. Thus, chitosan/interfering RNA gene targeting technology is a valuable laboratory technique with potential for broader applications. Future research will explore additional applications for this technique. Moreover, chemical modification of chitosan and other polymers, an active area of research, may increase the efficiency of interfering RNA delivery or permit targeted delivery to specific tissues ${ }^{37}$.

\section{Disclosures}

The authors have nothing to disclose.

\section{Acknowledgements}

Funding from the Kansas Agricultural Experiment Station and K-State Arthropod Genomics Center to KYZ supported the original development of chitosan/dsRNA-mediated gene silencing in A. gambiae ${ }^{19}$. The A. gambiae work described here was supported in part by R01-Al095842 from NIH/NIAID to KM. Development of chitosan/siRNA-mediated gene silencing in A. aegypti ${ }^{20,21}$ was supported by NIH/NIAID Award R01-AI081795 to MDS. The contents of this study are solely the responsibility of the authors and do not necessarily represent the official views of the $\mathrm{NIH}$.

\section{References}

1. Malaria. World Health Organization http://www.who.int/topics/malaria/en (2014).

2. Dengue. MalariaCenters for Disease Control and Prevention http://www.cdc.gov/dengue/ (2014).

3. Knipling, E. F., et al. Genetic control of insects of public health importance. Bulletin of the World Health Organization. 38, (3), 421-438 (1968).

4. Fu, G., et al. Female-specific flightless phenotype for mosquito control. Proceedings of the National Academy of Sciences of the United States of America. 107, (10), 4550-4554 (2010).

5. Wise de Valdez, M. R., et al. Genetic elimination of dengue vector mosquitoes. Proceedings of the National Academy of Sciences of the United States of America. 108, (12), 4772-4775 (2011).

6. Harris, A. F., et al. Successful suppression of a field mosquito population by sustained release of engineered male mosquitoes. Nature Biotechnology. 30, (9), 828-830 (2012).

7. Fire, A., et al. Potent and specific genetic interference by double-stranded RNA in Caenorhabditis elegans. Nature. 391, (6669), 806-811 (1998).

8. Yu, N., et al. Delivery of dsRNA for RNAi in insects: an overview and future directions. Insect science. 20, (1), 4-14 (2013).

9. Zhang, H., Li, H. C., Feasibility Miao, X. X. limitation and possible solutions of RNAi-based technology for insect pest control. Insect science. 20, (1), 15-30 (2013).

10. Clemons, A., Haugen, M., Severson, D. W., Duman-Scheel, M. Functional analysis of genes in Aedes aegypti embryos. Cold Spring Harb Protoc. 2010, (10), (2010).

11. Clemons, A., et al. siRNA-mediated gene targeting in Aedes aegypti embryos reveals that frazzled regulates vector mosquito CNS development. PLoS One. 6, (1), e16730 (2011).

12. Haugen, M., et al. Semaphorin-1a is required for Aedes aegypti embryonic nerve cord development. PLoS One. 6, (6), e21694 (2011).

13. Nguyen, C., et al. Functional genetic characterization of salivary gland development in Aedes aegypti. EvoDevo. 4, (1), 9 (2013).

14. Sarro, J., et al. Requirement for commissureless2 function during dipteran insect nerve cord development. Developmental dynamics: an official publication of the American Association of Anatomists. 242, (12), 1466-1477 (2013).

15. Singh, A. D., Wong, S., Ryan, C. P., Whyard, S. Oral delivery of double-stranded RNA in larvae of the yellow fever mosquito, Aedes aegypti: implications for pest mosquito control. J Insect Sci. 13, 69 (2013).

16. Lopez-Martinez, G., Meuti, M., Denlinger, D. L. Rehydration driven RNAi: a novel approach for effectively delivering dsRNA to mosquito larvae. Journal of medical entomology. 49, (1), 215-218 (2012).

17. Scott, J. G., et al. Towards the elements of successful insect RNAi. Journal of insect physiology. 59, (12), 1212-1221 (2013).

18. Coy, M. R., et al. Gene silencing in adult Aedes aegypti mosquitoes through oral delivery of double-stranded RNA. J Appl Entomol. 136, (10), 741-748 (2012).

19. Zhang, X., Zhang, J., Zhu, K. Y. Chitosan/double-stranded RNA nanoparticle-mediated RNA interference to silence chitin synthase genes through larval feeding in the African malaria mosquito (Anopheles gambiae). Insect molecular biology. 19, (5), 683-693 (2010).

20. Mysore, K., Flannery, E. M., Tomchaney, M., Severson, D. W., Duman-Scheel, M. Disruption of Aedes aegypti olfactory system development through chitosan/siRNA nanoparticle targeting of semaphorin-1a. PLoS neglected tropical diseases. 7, (5), e2215 (2013).

21. Mysore, K., Andrews, E., Li, P., Duman-Scheel, M. Chitosan/siRNA nanoparticle targeting demonstrates a requirement for single-minded during larval and pupal olfactory system development of the vector mosquito Aedes aegypti. BMC developmental biology. 14, (1), 9 (2014).

22. Dass, C. R., Choong, P. F. Chitosan-mediated orally delivered nucleic acids: a gutful of gene therapy. Journal of drug targeting. 16, (4), 257-261 (2008).

23. An, C., Budd, A., Kanost, M. R., Michel, K. Characterization of a regulatory unit that controls melanization and affects longevity of mosquitoes. Cellular and molecular life sciences: CMLS. 68, (11), 1929-1939 (2011).

24. Clemons, A., Mori, A., Haugen, M., Severson, D. W., Duman-Scheel, M. Culturing and egg collection of Aedes aegypti. Cold Spring Harb Protoc. 2010, (10), (2010). 
25. Horn, T., Boutros, M. E-RNAi: a web application for the multi-species design of RNAi reagents--2010 update. Nucleic acids research. 38 , W332-W339 (2010).

26. Patel, N. H. Ch. 19. In situ hybridization to whole mount Drosophila embryos. A laboratory guide to RNA: Isolation, Analysis, and Synthesis. PA, K. rieg Wiley-Liss (1996).

27. Haugen, M., et al. Whole-mount in situ hybridization for analysis of gene expression during Aedes aegypti development. 2010, (10), Cold Spring Harb Protoc (2010).

28. Clemons, A., Flannery, E., Kast, K., Severson, D. W., Duman-Scheel, M. Immunohistochemical analysis of protein expression during Aedes aegypti development. Cold Spring Harb Protoc. 2010, (10), (1101).

29. Rund, S. S., Hou, T. Y., Ward, S. M., Collins, F. H., Duffield, G. E. Genome-wide profiling of diel and circadian gene expression in the malaria vector Anopheles gambiae. Proceedings of the National Academy of Sciences of the United States of America. 108, (32), E421-E430 (2011).

30. Gaj, T., Gersbach, C. A., Barbas, C. F. 3rd ZFN, TALEN, CRISPR/Cas-based methods for genome engineering. Trends in biotechnology. 31, (7), 397-405 (2013)

31. Aryan, A., Myles, K. M., Adelman, Z. N. Targeted genome editing in Aedes aegypti using TALENs. Methods. 69, (1), 38-45 (2014).

32. Sarathi, M., Simon, M. C., Venkatesan, C., Hameed, A. S. Oral administration of bacterially expressed VP28dsRNA to protect Penaeus monodon from white spot syndrome virus. Mar Biotechnol (NY). 10, (3), 242-249 (2008).

33. He, B., et al. Fluorescent nanoparticle delivered dsRNA toward genetic control of insect pests). Adv Mater. 25, (33), 4580-4584 (2013).

34. Giljohann, D. A., Seferos, D. S., Prigodich, A. E., Patel, P. C., Mirkin, C. A. Gene regulation with polyvalent siRNA-nanoparticle conjugates. Journal of the American Chemical Society. 131, (6), 2072-2073 (2009).

35. Davis, M. E., et al. Evidence of RNAi in humans from systemically administered siRNA via targeted nanoparticles. Nature. 464, (7921), 1067-1070 (2010).

36. Molinaro, R., et al. Polyethylenimine and chitosan carriers for the delivery of RNA interference effectors. Expert opinion on drug delivery. 10, (12), 1653-1668 (2013).

37. Singha, K., et al. Polymers in small-interfering RNA delivery. Nucleic acid therapeutics. 21, (3), 133-148 (2011). 